\title{
ESTABLISHING IMMUNIZATION SAFETY SURVEILLANCE SYSTEM IN ALBANIA
}

\author{
Irsida Mehmeti ${ }^{1}$, Silva Bino ${ }^{2}$
}

\begin{abstract}
INTRODUCTION: While vaccine use increases, adverse events following immunization are becoming a public concern with the rate of adverse events following immunization (AEFI) increasing with the number of administered doses of vaccines. The safety of vaccines is ensured during clinical studies before the licensure of the vaccine. However, clinical trials have limitations, such as, rare events that cannot be detected and events occurring in specific groups of people. The consequences of AEFI influence public health policies and the incidence of vaccine-preventable diseases, although these might not be related directly to vaccines themselves.
\end{abstract}

OBJECTIVES: The objective of this article is to examine the evolution of vaccine safety issues in Albania by mapping stakeholders involved in vaccine safety control and analyzing the different periods when changes in the vaccine safety system has taken place

METHODS: A grey literature review, by searching the archive of IPH, provided a list of different stakeholders involved in vaccine safety in Albania. By reviewing all documents of the various organizations included in vaccine safety, we differentiated and characterized the roles of each in this area.

RESULTS: The main stakeholders involved in AEFI and vaccine safety in Albania are; the Institute of Public Health (IPH), which manages the National Immunization Program (NIP), the Directorate of Public Health (DPH) at the Ministry of Health (MoH), the 36 District Public Health Directories each with a microbiological laboratory for the assessment of AEFI, the pharmacovigilance center and National Regulatory Authority in the National Agency for Medicines and Medical Devices (NAMMD), National Immunization Safety Expert Committee in $\mathrm{MoH}$ and the Secretariat that supports the national AEFI committee

CONCLUSIONS: Despite huge progress over the last decade in Albania regarding vaccine safety, much remains to be achieved. The changes in the vaccine safety system have enforced and increased public trust on vaccination, leading to a strengthened National Immunization Program.

UDC Classification: 614.4, 614.3, 614.39 DOI: http://dx.doi.org/10.12955/cbup.v4.824

Keywords: Vaccine safety, immunization, surveillance, adverse events.

\section{Introduction}

Albania has a strong immunization program, with routine immunization coverage estimated as over 98\% for all antigens (World Health Organization \& UNICEF, 2015). Although vaccines are generally safe and cost-effective in preventing many diseases, safety measures are needed to assure the continuous success of immunization program (Duclos, 2004). Internal migration, immigration, urbanization, and reforms within the health care system have influenced the immunization system, vaccine coverage rates, and vaccine safety issues in Albania. The vaccination program was established in the 1960s. Before 1990, most of the vaccines of the national immunization schedule were produced in Albania, apart from the oral polio virus (OPV), which was imported from abroad to cover all of the country's needs. In 1993, the Ministry of Health in collaboration with Institute of Public Health (IPH) set up the Expanded Program on Immunization (EPI), in accordance with the World Health Organization (WHO) requisites and objectives (World Health Organization \& PATH, 2015). The objectives of the EPI comprise the continuous surveillance of vaccine preventable diseases and monitoring of vaccination coverage, as well as ensuring vaccine safety.

Albania is divided administratively into 36 districts. Each district has its own epidemiological laboratory where reported adverse events following immunization (AEFI) can be investigated. Dedicated vaccination staff exist at the district level and in the primary health care centers. Immunization service providers in Albania include maternity hospitals and child consultancies

\footnotetext{
${ }^{1}$ Irsida Mehmeti, Department of Pharmaceutical Sciences, Catholic University "Our Lady of Good Counsel", Tirana, Albania, irsida.mehmeti@gmail.com

${ }^{2}$ Silva Bino, Control of Infectious Diseases Department, Institute of Public Health, Tirana, Albania
} 
situated at primary health care services in urban areas and rural areas. All the vaccines of the immunization schedule are prequalified from standards of $\mathrm{WHO}$ and meet the criteria of legislation on medicines (WHO, 2015).

Albania has introduced six new vaccines into the routine immunization program over the past 15 years. When a new vaccine is being considered for introduction in Albania, an evidence-based analysis is conducted to determine whether or not the evidence supports the vaccine introduction (Johns Hopkins Bloomberg School of Public Health \& International Vaccine Access Center,IVAC, 2015, p. 9).

The World Health Organization (WHO, 2014) defines an AEFI as a medical incident that takes place after an immunization, causes concern, and is believed to be caused by immunization. Although data of preclinical and premarketing studies have shown and guaranteed the safety of vaccines, there are some events that are detected only after the product has been launched for marketing. These events include those that occur rarely and those that occur in specific groups of people who are not represented in clinical trials. In some cases, these reactions are caused by the vaccine; in others, they are caused by administration error or handling of the vaccine; and in most cases, the event is related to vaccination only by time proximity. Whatever the cause, when an adverse event following an immunization (AEFI) raises concerns among the public to the extent that they refuse further immunizations for their children, the children are much more likely to contract and then suffer the consequences of a vaccine-preventable disease (Food, Medicine and Healthcare Administration and Control Authority of Ethiopia, 2011, p. 7).

The importance of establishing an immunization safety surveillance is closely related to vaccine coverage rates and an increased trust in vaccination. An efficient post-marketing surveillance system for vaccines becomes a key tool to strengthen the immunization system where performance indicators are coverage rate and the percentage of people participating in the immunization system.

The AEFI reporting system in Albania started in 2001 with the establishment of the National Regulatory Authority of Vaccines in the IPH. Before 2000, the reporting of any adverse event was ad hoc within the national immunization program, as all vaccines were purchased through UNICEF (Bino, 2003). The National Regulatory Authority serves as a national focal point and resource center for pharmacovigilance activities, along with the Pharmacovigilance Department in the National Agency of Medicines and Medical Devices (NAMMD; Kucuku, 2012). In Albania, the AEFI reporting is mandatory by law. Setting up awareness on the importance and relevance of the reporting of adverse events following immunization and the vaccine safety from monitoring was important for sustaining the national immunization program (NIP) and increasing vaccine coverage. Among the tools designed by the Institute of Public Health and National Agency of Medicines and Medical Devices is an AEFI reporting form, along with a guideline on how to report adverse events and distribute the report to health facilities in the country. The center, in collaboration with IPH, also provides technical advice that helps the local authorities improve the AEFI management from time to time.

In 2004, WHO launched the Developing Countries Vaccine Regulators Network. The purpose of this network was to increase and strengthen the regulatory capacity of developing countries (WHO, 2011). Member countries may share expertise and information about problems of vaccine safety and efficacy. Member countries, including Albania, started to submit data on adverse events regarding medicines and later, data regarding adverse events following immunization, to a common database housed at the Uppsala Monitoring Centre, a WHO Collaborating Centre in Sweden.

The AEFI surveillance should be developed by different systems and bodies with well-defined and complementary roles. The NIP and National Regulatory Authority (NRA) could collaborate, as both parties are responsible for the safety of vaccines. The most important performance indicator of a wellestablished AEFI reporting system is high level of appropriate and timely reporting.

\section{Provisions Undertaken in Albania to Ensure Vaccine Safety}

According to the 2011 revision of the existing classification regarding cause-specific categorization of AEFI, by WHO and the Council for International Organizations of Medical Sciences (World Health Organization \& CIOMS; 2012, p. 41), an AEFI may be related to one or more of the following causes:

1. Vaccine-product related reaction; 
2. Vaccine-quality defect related reaction;

3. Immunization-error related reaction;

4. Immunization anxiety related reaction; and

5. Coincidental event.

The safety of vaccines should be assured, starting with their production and continuing through manufacturing, packaging, transport, storage, until the administration of the vaccine to the individual (World Health Organization, 2011). Establishing a surveillance system to control the safety of vaccines is not just a temporary challenge, but rather requires a continuous effort and a well-planned project (World Health Organization, 2014).

Albania has launched several initiatives to strengthen vaccine safety in recent years, starting from the assurance of a secure delivery to prevent immunization error related to reactions, which are the most common cause of adverse events following immunization, continuing with collaboration with reliable sources to procure safe vaccines, and onto establishing regulatory bodies that oversee and supervise safety issues. These include the following:

- The first efforts to assure the safety of vaccination started since 1993 when auto-disabled syringes (AD) were introduced in Albania (Safe Injection Global Network, 2001). Previously, all immunization practices involved reusable syringes, which were autoclaved in health care centers. The introduction of safe syringes avoided infection from microbial contaminant at the site of injection during the administration of the vaccine, an event classified as immunization error related.

- Improving and managing logistic problems regarding poor vaccine stock, handling, and storage avoids immunization error related reactions. Recording immunization data in an online immunization system enables better management of vaccine stock (Safe Injection Global Network, 2001). Storage of vaccines for lengthy periods alters their quality, increasing the risk for an adverse reaction at injection. An online system that manages information about the immunization status of children, vaccine stock, adverse events following immunization (AEFI), and cold chain conditions at every location has the potential to improve the reporting of AEFI, identify clusters on time, and take appropriate actions (World Health Organization \& PATH, 2015, p.10)

- Implementing specific vaccine procurement strategies provides vaccines from well-qualified sources. All vaccines included in NIP are procured through UNICEEF, which supply vaccines from only prequalified manufacturers. Through a process of pre-qualification, WHO advises UN procurement agencies of the quality, efficacy, and safety of vaccines available on the market (Bino \& Ahmetaj, 2015).

- Cold chain management is another factor that influences the quality of vaccines. Because of the temperature-sensitivity of vaccines, special attention should be paid to all steps during transport and storage of vaccines (Kartoglu \& Milstien, 2014). The inspection of vaccines on arrival at cold stores is carried out to assure the quality of vaccines at point of delivery, the proper recording of vaccine identification (type, manufacturer, batch, and expiry), and provide indicators for monitoring performance of vaccine deliveries, transport, and storage including the time of administration (UNICEF, 2002, p. 1). The Vaccine Vial Monitor (VVM) shows whether the vaccine has previously been exposed to temperatures that might have compromised the quality of the vaccine. In Albania, VVM was introduced in 1996 for the vials of OPV vaccine and later for all types of vaccines. Previously, vaccine quality was tested only through laboratorys, which indicated whether a vaccine could be used or not and did not need electricity to function. This advantage made it an appropriate tool to ensure vaccine quality in areas difficult to reach and without electricity. After checking the status of temperature monitors, vial sizes, quantities, condition of boxes, and documentation arriving with the shipment, a Vaccine Arrival Report is completed (PATH, 2011). Additionally, many cold chain equipment units have been replaced, and since 2013, all cold chain units in the country are pre-qualified (Johns Hopkins Bloomberg School of Public Health \& International Vaccine Access Center, 2015, p. 8).

- The establishment of various important vaccine safety control organizations included the Pharmacovigilance Department in 2012 in the National Agency of Medicines and Medical 
Devices (Kucuku, 2012). This involved establishing a national (central) expert committee for causality assessment, high-level technical support, and dates for decision-making. The National Immunization Safety Expert Committee is the highest advisory organism to provide opinion to the $\mathrm{MOH}$ on assessment of adverse events following immunization and vaccine safety. In the same year, i.e., 2012, the National AEFI Secretariat was established to support the National AEFI committee.

\section{Methods}

A grey literature review, by searching the archive of IPH, provided a list of different stakeholders involved in vaccine safety in Albania. By reviewing all documents of the various organizations included in vaccine safety, we differentiated and characterized the roles of each in this area. In addition, through a published literature review, we identified the steps to detect and respond on time for a serious AEFI or one that would represent a relevant public concern in Albania.

\section{Results: Mapping Stakeholders of Immunization Safety in Albania and Their Roles}

In recent years, Albania has worked intensely to implement the objectives of the Global Vaccine Safety Blueprint, which is a strategic document developed by WHO (2011) to help low to middle income countries establish vaccine safety systems.

There immunization safety surveillance in Albania includes a range of governmental, nongovernmental, and commercial bodies at the following levels:

- national (central);

- subnational or intermediate (district); and

- service-provider level.

The following are the main stakeholders involved in AEFI and vaccine safety in Albania:

1. The Institute of Public Health (IPH), which represents the only national research-scientifictraining-reference-expertise center in the public health (Kakarriqi, 2002, p. 12). The IPH is under the administrative supervision of the Ministry of Health $(\mathrm{MoH})$ in Albania and functions as the technical and policy-maker organis $\mathrm{m}$ of $\mathrm{MoH}$. It is responsible for purchasing vaccines, injection safety equipment, and managing most of the cold chain. Immunization strategies are developed by the IPH and endorsed by MoH. The role and tasks of IPH at the district and regional level are performed by the district directorates of public health, as follows:

a. the National Immunization Program (NIP), part of the IPH Department of Epidemiology and Control of Infectious Diseases manages the implementation of the nationwide immunization program; and

b. the infectious diseases surveillance center performs the surveillance of vaccine preventable diseases and AEFI.

2. The Directorate of Public Health (DPH) at the Ministry of Health $(\mathrm{MoH})$ implements public health programs in the country and oversees district public health directories.

3. The District Public Health Directories has 36 districts, each with a microbiological laboratory for the assessment of AEFI. The district directories are responsible for planning, distributing, and overseeing the immunization program within their district (World Health Organization \& PATH, 2015).

4. The pharmacovigilance center and National Regulatory Authority in the National Agency for Medicines and Medical Devices (NAMMD) manages the vaccine safety procedures.

5. National Immunization Safety Expert Committee in $\mathrm{MoH}$ and the Secretariat that supports the national AEFI committee provides important guidance on the introduction of new vaccines, their effectiveness, impact, and safety based on the best evidence and current good practice and expert opinion.

6. Primary health care centers, of which there are about 2,282 child consultation services in urban health centers (including maternity hospitals) and in health posts in rural areas, provide immunization practice (World Health Organization \& PATH, 2015, p. 4) 
7. Hospitals: District and Regional Hospitals and Tirana University Hospital "Mother Theresa", which through pediatric departments follow and provide causality assessments.

\section{Flow of Information Related to AEFI}

The tools used for flow of information include AEFI reporting and investigation forms (from the IPH) and the conclusions of the AEFI committee. The exchange of information proceeds according to protocols developed by the IPH and NAMMD. The IPH is responsible for recording the AEFI to the Vigibase ${ }^{\circledR}$ database through a Vigiflow ${ }^{\mathrm{TM}}$ report. Printed copies of the uploaded AEFI Vigiflow reports are submitted to National Agency of Medicines and Medical Devices and MoH. The IPH is also responsible for preparing guidelines on AEFI, especially on causality assessments.

\section{Steps of AEFI Detection and Response in Albania}

Adverse events that follow immunization are detected mainly through passive surveillance systems using individual AEFI case reporting forms. Active surveillance is performed only for selected AEFI (Bino et al., 2003). The main objectives of immunization safety surveillance are to detect, on time, the AEFI in order to analyze and respond as soon as possible to public concern and manage the event.

For an effective immunization safety surveillance system, it is necessary to involve health workers at all levels of the immunization program (World Health Organization, 2013).

\section{Detection of AEFI}

The detection of AEFI is mainly performed by the public health sector, but recently the private sector has also been engaged. Detection of AEFI is the responsibility of the health workers providing immunization services in primary care or other facilities in private health care system; as well as health workers providing clinical treatment of AEFIs in health posts, health centers, hospitals, or special treatment facilities; and parents who suspect AEFIs in their children (Food, Medicine and Healthcare Administration and Control Authority of Ethiopia, 2011)

\section{Recording of AEFI}

The forms and registers necessary for immunization safety surveillance are supplied and maintained by the IPH and public health directories (Nelaj, Mesi, Preza, \& Xhabija, 2014). All necessary data should be entered into the forms and then, in the AEFI registers. At the national level, adverse event following immunization are recorded in a national database of AEFI using Microsoft Excel software.

\section{Reporting of AEFI}

The AEFI reporting form is completed by the Primary Health Care Center staff who sends it to the Epidemiologic Department of the DPH. The district Directorate of Public Health confirms the AEFI, assigns a code number, and completes the details missing in the reporting form within 24 hours. Personnel at the subnational level need to immediately inform those at the national level of any serious or unusual AEFI events (including deaths). Other cases are reported routinely. At an international level the AEFI cases are reported routinely through VigiFlow on an individual case report form. VigiFlow is a web-based Individual Case Safety Report (ICSR) management system that is specially designed for use by national centers in the WHO Program for International Drug Monitoring ${ }^{3}$. A standardized case definition checklist, which has adopted Brighton Collaboration case definitions, is distributed to all health care units ${ }^{4}$. Where there is a necessity for treatment of a particular condition, the recipient undergoing an AEFI is referred to the nearest hospital, health facility, or the Tirana University Hospital Centre. The AEFI data are also reported annually to WHO through the joint reporting form (JRF) to World Health Organization and UNICEF (WHO \& UNICEF, 2015). Using the JRF allows the monitoring of immunization safety performance globally and continuously.

\footnotetext{
${ }^{3}$ For more details please see www.who-umc.org

${ }^{4}$ For more details please see https://brightoncollaboration.org/public
} 
Figure 1: The scheme explains the way in which adverse events following immunization (AEFI) information is reported and shared between various stakeholders in Albania

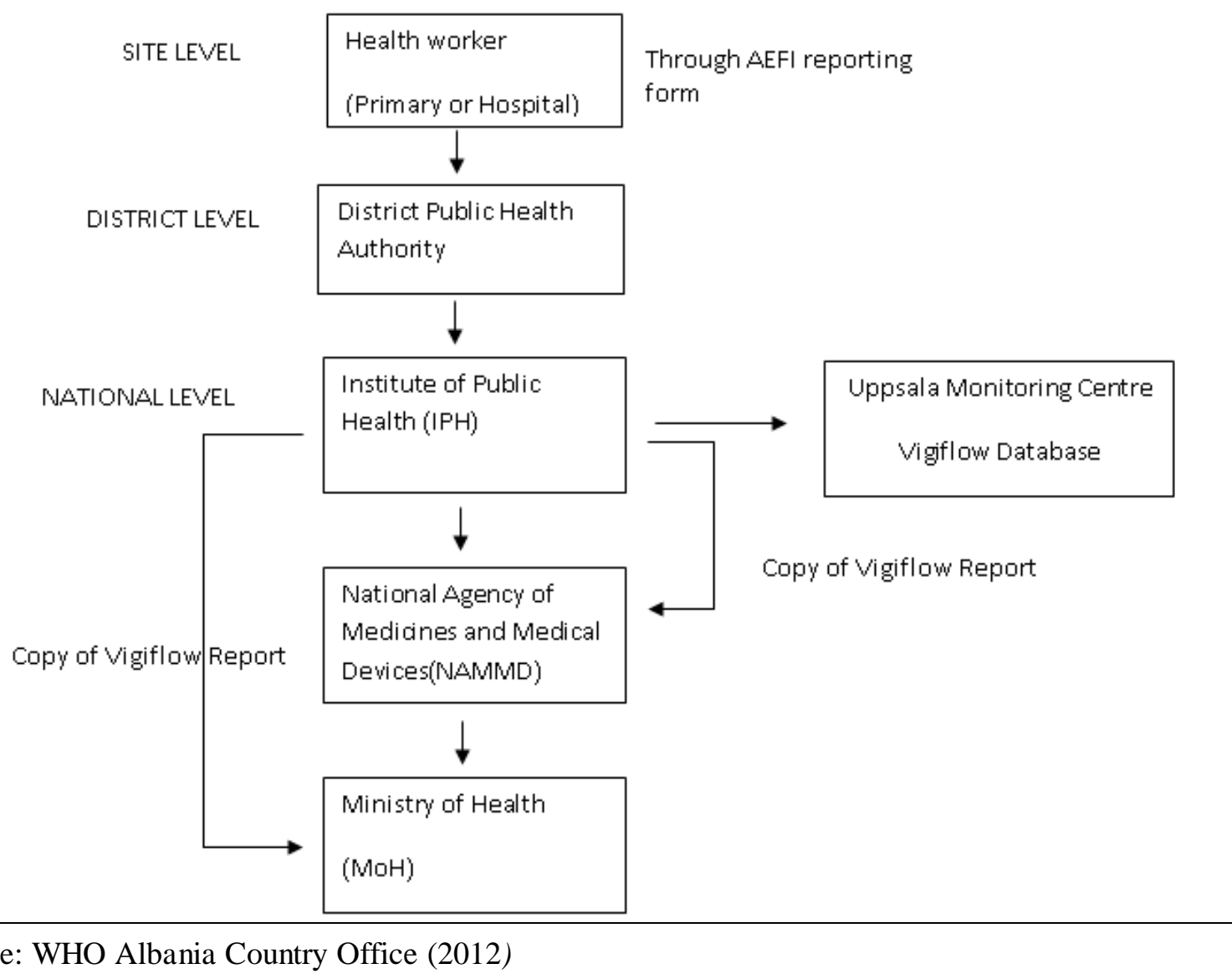

\section{Investigation of AEFI}

The report of an AEFI is followed by a case investigation to establish the cause of an AEFI or cluster of AEFI and treat such (Food, Medicine and Healthcare Administration and Control Authority of Ethiopia, 2011). The investigation is conducted at different levels and as soon as possible where required. Where the investigation is done by $\mathrm{DPH}$, the investigation form needs to be completed within seven days from the receipt of the AEFI reporting. When the AEFI is serious it should be treated with urgency and transferred to the IPH, whereas other investigations are sent normally, i.e., at pre-stabilized frequencies. The IPH should investigate for similar cases as soon as it receives any AEFI report from the district level. Investigations are appropriately supported with laboratory testing where necessary.

\section{Analysis of AEFI}

Analysis of data collected through the investigation form is crucial. Using AEFI reporting and investigation forms allow the IPH to create a line list and a national database through for analysis of data. After an analysis of data is completed, a report is built on the outcomes of the data analysis. The IPH analyses the investigation form received at the district level, consults with the AEFI committee and where needed, sends the investigation form to the NAMMD.

\section{Causality assessment}

A preliminary causality assessment can be carried out at the subnational level. Serious AEFI causality determination should be done by the IPH in collaboration with the pediatric department at the Tirana University Hospital. Those cases with pending conclusions are referred to the national AEFI Expert Committee for further evaluation and final classification (Maure, Dodoo, Bonhoeffer, \& Zuber, 2014). 


\section{Corrective and Preventive Actions}

As soon as the causality of an AEFI has been determined, action is taken to either cure it or prevent its further dissemination. Both corrective and preventive actions should be taken as early as possible (World Health Organization, 2014, p. 34). Corrective actions should be taken at both the subnational (district public health directorates) and the national level whenever necessary.

\section{Monitoring, Supervision, and Training}

Although monitoring, supervision, and training are the responsibility of the subnational level, the national level can assist with guidelines, training materials, and standard formats for supervision (World Health Organization, 2014). Staff at all levels should be trained in diagnosing, treating, and reporting AEFIs to avoid underreporting. In November 2011, a team comprising one WHO staff and one Vigiflow expert developed a training module in Albania about strengthening, monitoring, and causality assessment of adverse events following immunization. The training was helpful for all stakeholders involved in vaccine safety and helped in building capacity for vaccine surveillance, leading to better vaccine safety. The IPH and NAMMD jointly developed training materials through a core group of trainers.

\section{Public Education/Communication}

The conclusions and results of case investigations should be shared with public and health care workers, especially with parents to inform them about the advantages of vaccination (UNICEF, 2005).

\section{Conclusions}

Despite huge progress over the last decade in Albania regarding vaccine safety, much remains to be achieved. Albania has attained the first goal set by Global Vaccine Safety Blueprint and established a minimal capacity for vaccine safety control. The objectives for the future involve the remaining two goals of the Global Vaccine Safety Blueprint. This implies building an enhanced capacity to evaluate the safety of vaccines. Spontaneous reporting forms alone limit timely assessment and treatment of AEFI. Responding on time to a vaccine safety issue means preventing the emerging of the event where it is serious or preventing the 'dropping out' of the population from an immunization program because of an event unrelated to a vaccine. This is especially important in cases of new vaccines. Enhanced capacity includes the ability to set up active surveillance on the AEFI and developing a well-designed epidemiological study to investigate the cause of the AEFI. Nevertheless, there is no protocol that regulates and guides the investigation process and all investigation steps are electronically registered. The NAMMD should develop a protocol that defines the objectives for immunization safety surveillance, strategies, structure, activities, and resources. It is also necessary to strengthen the reporting system for the private health sector, which is not fully involved in recording such events.

All stakeholders included in vaccine safety should further clarify and agree on each of their roles and responsibilities. More collaboration and sharing of information is needed among these stakeholders through new digital technologies to avail the AEFI data for the regulators and other data users. From the review of all data and documents, it is concluded that the IPH continues to carry many responsibilities related to vaccine safety, while National Agency of Medicines and Medical Devices has yet to take on many responsibilities. The legal framework for a compensation scheme or social support scheme also remains lacking in Albania.

\section{References}

Bino, S. \& Ahmetaj, A. (2015). Vaccine Procurement and Regulations in Albania. Country Presentation. Retrieved January 12, 2016, from UNICEF website: http://www.unicef.org/supply/files/ALB_Country_Presentation_27_10_2015_rev1.pdf

Bino, S. (2003). Measles-Rubella Mass Immunization Campaign in Albania, November 2000. The Journal of Infectious Diseases J INFECT DIS, 187 (S1).

Duclos, P. (2004). A global perspective on vaccine safety. Vaccine, 22(15-16), 2059-2063.

Food, Medicine and Healthcare Administration and Control Authority of Ethiopia. (2011). Guide for AEFI Surveillance in Ethiopia. Retrieved October, 2015, from www.fmhaca.gov.et

Johns Hopkins Bloomberg School of Public Health \& International Vaccine Access Center (IVAC). (2015). Case Studies of IPV Introductions: Albania, Nigeria, and Tunisia. Baltimore. 
Kakarriqi E. (2002). Epidemiological background of infectious diseases in Albania (1960-2001) and their prevention and control in the context of natural disasters and infectious diseases. Albania, Tirana: Department of Epidemiology Institute of Public Health. Retrieved January 23, 2016, from http://www.gideononline.com/features/resources/

Kartoglu, U., \& Milstien, J. (2014). Tools and approaches to ensure quality of vaccines throughout the cold chain. Expert Review of Vaccines, 13(7), 843-854

Kucuku, M. (2012). Role of Pharmacovigilance on Vaccines Control. Journal of Rural Medicine J Rural Med,7(1), 42-45

Maure, C. G., Dodoo, A. N., Bonhoeffer, J., \& Zuber, P. L. (2014). The Global Vaccine Safety Initiative: Enhancing vaccine pharmacovigilance capacity at country level. Bulletin of the World Health Organization 92(9), 695-696.

Nelaj E., Mesi A., N., Preza I., \& Xhabija A. (2014). On the Electronic Vaccination System and its Advantages. Health Bulletin, 1(2), 11-16. Retrieved December 12, 2015, from http://www.ishp.gov.al/wp-content/uploads/2014/04/Bulletin20141-2.pdf

PATH. (2011). Assessment of a Remote Alarm System for Vaccine Storage in Albania. Retrieved September, 2015, from PATH website: http://www.path.org/publications/detail.php?i=2058

Safe Injection Global Network. (2001). Albania seizes an opportunity to improve injection safety. Retrieved fromhttp://www.injectionsafety.org/html/april_2001.html

UNICEF. (2005). Building trust and responding to adverse events following immunization in south Asia: using strategic communication. Retrieved February 10, 2016, from UNICEF website:

http://www.unicef.org/rosa/Immunisation_report_17May_05(final_editing_text).pdf

UNICEF. (2002). Guidelines for the use of the Vaccine Arrival Report in UNICEF shipments Retrieved February 1, 2016, from http://www.unicef.org/supply/index_vaccine_safety.html

World Health Organization \& PATH. (2015). Optimize: Albania Report. Seattle: PATH. Retrieved February, 2016, from PATH website: https://www.path.org/publications/files/TS_opt_albania_rpt.pdf

World Health Organization \& UNICEF. (2015). Albania: WHO and UNICEF estimates of immunization coverage. Retrieved October 10, 2015, from: http://www.who.int/immunization/monitoring_surveillance/data/alb.pdf

World Health Organization, Albania Country Office. (2012). [Draft report of the planning group meeting to chart out an action plan for Post Marketing Surveillance of AEFI]. Unpublished raw data.

World Health Organization. (2011). Global Vaccine Safety Blueprint. Retrieved January 10, 2016, from: http://www.who.int/immunization/sage/Blueprint_draft_14Oct2011_nov11.pdf

World Health Organization \& CIOMS. (2012). Definition and application of terms for vaccine pharmacovigilance. Report of the CIOMS/WHO Working Group on Vaccine Pharmacovigilance. Retrieved December, 2015, from World Health

Organization website: http://whqlibdoc.who.int/publications/2012/9789290360834_eng.pdf

World Health Organization. (2013). Causality assessment of an adverse events following immunization. User manual for the revised WHO classification. Retrieved February 1, 2016, from http://www.who.int/vaccine_safety/publications/aevi_manual.pdf

World Health Organization. (2014). Global manual on surveillance of adverse events following immunization. Retrieved October, 2015 from World Health Organization website:

http://www.who.int/vaccine_safety/publications/Global_Manual_on_Surveillance_of_AEFI.pdf 